\title{
Aplicando Redes Sociais Veiculares para Aprimorar o Gerenciamento da Mobilidade Urbana
}

\author{
Ademar T. Akabane, Roger Immich, \\ Edmundo R. M. Madeira, Leandro A. Villas \\ ${ }^{1}$ Instituto de Computação - Universidade de Campinas (UNICAMP), Brasil \\ takeodlrc.ic.unicamp.br, \{roger, edmundo, leandro\}@ic.unicamp.br
}

\begin{abstract}
The Advanced Traffic Management System (ATMS) has been increasingly used by urban mobility managers to improve vehicular traffic management. Many ATMSs employ centralized solutions because of the difficulty of selecting the most relevant vehicles, in highly dynamic networks, to detect congestion and suggest alternative routes. Furthermore, such solutions are not always scalable. On the other hand, the distributed solution needs to previously segment the entire scenario to select the vehicles. Moreover, such a solution suggests alternative routes, in a selfish fashion, which can lead to secondary congestions. Based on the found open issues, this work proposes a distributed urban mobility management system based on the vehicular social networks paradigm (VSNs) named MAESTRO. The VSNs paradigm emerged from the integration of intelligent wireless communication devices and social networks in the vehicular environment. Two different approaches can be explored in VSNs, i.e., the Social network analysis (SNA) and the Social Network Concepts (SNC). The proposed MAESTRO system adopts a combined use of both SNA and SNC approaches. Simulation results showed that the use of SNA and SNC, in a vehicular environment, has great potential in increasing the scalability of the system and also improving efficiency in the management of urban mobility.
\end{abstract}

Resumo. O Sistema Avançado de Gerenciamento de Tráfego (ATMS) é cada vez mais utilizado por gestores de mobilidade urbana para aprimorar o gerenciamento de tráfego veicular. Muitos ATMSs empregam soluções centralizadas devido à dificuldade de selecionar os veículos mais relevantes, em redes altamente dinâmicas, para detectar congestionamentos e sugerir rotas alternativas. Além disso, tais soluções nem sempre são escaláveis. Por outro lado, a solução distribuída necessita previamente segmentar todo o cenário para selecionar os veículos. Além disso, tal solução sugere rotas alternativas, de maneira egoísta, que pode levar ao congestionamento secundário. Com base nessas lacunas encontradas, foi proposto um sistema distribuído de gerenciamento de mobilidade urbana baseado no paradigma das redes sociais veiculares (VSNs) chamado de MAESTRO. Tal paradigma surgiu a partir da integração dos dispositivos de comunicação sem-fio e das redes sociais no ambiente veicular. Assim duas diferentes abordagens podem ser exploradas em VSNs: Social Network Analysis (SNA) e Social Network Concepts (SNC). Ambas abordagens foram aplicadas no sistema MAESTRO. Os resultados das simulações mostraram que o uso dos SNA e SNC, no ambiente veicular, tem grande potencial em aumentar a escalabilidade do sistema e também aprimorar a eficiência no gerenciamento da mobilidade urbana.

\section{Introdução}

O paradigma das redes sociais veiculares (VSNs) surgiu através da integração dos conceitos de redes sociais móveis com as redes ad-hoc veiculares (VANETs) [Vegni and Loscri 2015, Qin et al. 2016, Rahim et al. 2017]. Partindo desse 
princípio, duas abordagens principais sobre tal paradigma podem ser exploradas no cenário veicular: (i) aplicação das técnicas de Social Network Analysis (SNA) [Qin et al. 2016, Rahim et al. 2017] e/ou (ii) utilização dos Social Network Concepts (SNC) [Vegni and Loscri 2015, Rahim et al. 2017]. A primeira abordagem concentra-se em identificar a importância do nó na rede. Para este fim, as três principais medidas de centralidade mais aplicadas em VSNs são: grau, proximidade e intermediação [Qin et al. 2016, Rahim et al. 2017]. Sabe-se que em VSNs a topologia da rede é altamente dinâmica, logo calcular a centralidade do nó é uma tarefa desafiadora. Por outro lado, uma vez identificada, pode ser útil para diversas aplicações, como o gerenciamento do fluxo de informações dentro da rede. Já as SNC envolvem interações sociais entre os nós que possuem interesses mútuos dentro da comunidade virtual temporal [Vegni and Loscri 2015, Akabane et al. 2018b]. Em outras palavras, nessa abordagem é dada a oportunidade dos veículos participarem de uma comunidade virtual veicular e compartilharem as informações de interesse mútuo por meio das interações sociais. Partindo dessa ideia, cada motorista pode compartilhar sua informação social, por exemplo, a rota pessoal. Dessa forma, permitindo a prática do planejamento de rota colaborativo. A interação social é dada no momento em que os veículos se encontram e compartilham suas informações sociais uns com os outros por meio de canais de comunicação sem fio.

ATMS integra as tecnologias de comunicação, armazenamento e processamento com intuito de coletar dados brutos, provenientes das VSNs, e extrair conhecimentos relacionados ao trânsito [Akabane et al. 2018b]. Por meio do conhecimento gerado, os ATMSs podem prover serviços que melhoram a eficiência do gerenciamento de tráfego veicular. Além disso, muitas aplicações de ATMS necessitam que os veículos periodicamente compartilhem os seus dados (floating car data) entre os veículos vizinhos ou com o servidor central e roadside unit (RSU). Através dessa operação é possível criar o conhecimento sobre as condições de tráfego veicular [Wang et al. 2016, Doolan and Muntean 2017, Pan et al. 2017]. Essa prática é conhecida como beaconing e os dados trocados estão relacionados com a mobilidade do veículo tais como identificação do veículo, posição atual do veículo, velocidade, direção de deslocamento, entre outros.

Diferentes ATMSs foram projetados e implementados para superar o problema da baixa mobilidade urbana por meio do gerenciamento do tráfego veicular [Wang et al. 2016, de Souza and Villas 2016, Doolan and Muntean 2017, Pan et al. 2017]. As soluções de [Doolan and Muntean 2017, Pan et al. 2017] aplicam abordagem centralizada devido à dificuldade de selecionar os veículos mais relevantes, em redes altamente dinâmicas, para as tarefas de detecção de congestionamento e sugestão de rotas alternativas. Além do mais, tais soluções esbarram na dificuldade de escalar os sistemas. A solução de [de Souza and Villas 2016] emprega uma abordagem distribuída para detecção de congestionamento e sugestão de rotas. Porém, necessita previamente segmentar todo o cenário em várias subregiões para atingir seu objetivo. Além do mais, a rota é calculada de forma egoísta, sem considerar as rotas escolhidas pelos veículos da vizinhança, o que pode acarretar congestionamentos secundários.

Com base nas lacunas encontradas, foi projetado e implementado o MAESTRO, um sistema distribuído de gerenciamento de mobilidade urbana baseado em VSNs. Inspirado nas duas abordagens das VSNs anteriormente mencionadas, uma técnica SNA é aplicada para classificar e selecionar os veículos em cada agrupamento (clustering), com intuito de reduzir o consumo da largura de banda. Enquanto que os SNC foram aplicados para realizar a troca de informações de interesse em comum, por meio da interação social, dentro de uma comunidade virtual temporal. Essa troca de informação auxilia no planejamento da rota alternativa de forma colaborativa, melhorando assim o gerenciamento da mobilidade urbana. 
De forma resumida, o foco do sistema MAESTRO é minimizar os problemas causados pelo congestionamento de tráfego veicular de forma distribuída e sem comprometer a sua escalabilidade. A avaliação do sistema proposto foi realizada por meio de simulações comparando com outros sistemas da literatura [de Souza and Villas 2016, Doolan and Muntean 2017, Pan et al. 2017] em diferentes requisitos. As avaliações de desempenho foram feitas sobre duas perspectivas: (i) avaliação de escalabilidade e (ii) avaliação do gerenciamento da mobilidade urbana.

O restante deste artigo está organizado da seguinte maneira. Na Seção 2 é descrita uma visão geral dos trabalhos existentes na literatura para gerenciamento da mobilidade urbana. A solução proposta neste trabalho é apresentada na Seção 3 e as avaliações dos resultados dos experimentos são descritas na Seção 4. Por fim, na Seção 5 é apresentada a conclusão e o trabalho futuro.

\section{Trabalhos Relacionados}

Ao longo dos anos, diversos autores propuseram sistemas para gerenciamento da mobilidade urbana com base no paradigma das VANETs. Nesta seção são descritos alguns trabalhos relacionados que foram utilizados na avaliação comparativa com o sistema MAESTRO.

Por exemplo, [Doolan and Muntean 2017] propuseram um sistema centralizado de gerenciamento de tráfego veicular batizado de EcoTrec. O sistema é centralizado pois a detecção do congestionamento e o cálculo da rota alternativa são realizados por meio de uma entidade central. Ecotrec tem como objetivo diminuir as emissões de $\mathrm{CO}_{2}$ sem afetar significativamente o tempo de viagem. Para este fim, tal sistema foi construído sobre uma arquitetura de três componentes: Vehicle Model, Road Model e Traffic Model. O Vehicle Model coleta e atualiza as informações individuais do veículo, além de compartilhá-las periodicamente com o Road Model. Os conteúdos compartilhados são provenientes do GPS (Global Positioning System), acelerômetro e giroscópio embarcados nos veículos. Já o Road Model é mantido nas RSUs que estão ao longo das vias conectado pelo Traffic Model. O Traffic Model é um servidor central que contêm as características e as condições de tráfegos das vias. Cada veículo faz requisições periódicas ao servidor sobre a condição de tráfego da via. Caso seu trajeto encontra-se congestionado, o servidor envia uma rota alternativa. Observa-se que esta abordagem pode acarretar uma alta sobrecarga da rede durante as trocas de informações, especialmente nas condições de tráfego denso.

Outro exemplo, os autores de [Pan et al. 2017] propuseram um sistema de gerenciamento veicular urbano híbrido nomeado de DIVERT. É uma abordagem híbrida pois necessita de um servidor central para coletar informações provenientes dos veículos e detectar os congestionamentos. Já o cálculo das rotas alternativas é realizado pelos veículos de forma colaborativa. No sistema DIVERT, o servidor central opera como um coordenador que recebe as informações dos veículos (velocidade, localização e direção) por meio de comunicação V2I. Provido de tais informações o servidor é capaz de detectar locais congestionados e alertar os veículos que estão dirigindo para o local detectado. Nesse sistema a responsabilidade do cálculo das rotas alternativas é dada para os veículos. $\mathrm{O}$ veículo, que necessita computar uma rota alternativa, leva em consideração a rota escolhida dos veículos vizinhos, ou seja, aplica-se uma decisão de roteamento colaborativo. Vale ressaltar que, neste sistema, o mecanismo de supressão de broadcast não foi aplicado durante o processo de disseminação de mensagens. Consequentemente, aumentando o consumo da largura de banda da rede e assim comprometendo a escalabilidade do sistema.

No trabalho [de Souza and Villas 2016] foi proposto um sistema distribuído para o gerenciamento de tráfego veicular, nomeado de FASTER. Tal sistema é distribuído pois a identificação do congestionamento e o cálculo da rota alternativa não necessitam de 
uma infraestrutura. FASTER necessita previamente segmentar todo o cenário em várias subregiões (ou distritos) para agregar as informações do tráfego das vias. Cada distrito possui uma área igual à área de comunicação de 1-salto dos veículos. Cada veículo periodicamente coleta e transmite informações como velocidade média e identificação da via para todos que estão dentro do seu raio de comunicação. No momento que o veículo recebe informações das vias, este armazena essa informação em suas listas de conhecimento de tráfego. O veículo que estiver mais próximo ao centro do distrito é selecionado para iniciar a disseminação das informações de tráfego agregadas para outros veículos. Durante o processo de disseminação é aplicado um mecanismo de supressão de broadcast para evitar a sobrecarga da rede. Em tal sistema, o cálculo da rota alternativa é realizado de forma egoísta, com base no algoritmo de $k$-menores caminhos com escolha probabilística. Isto é, não é levada em consideração as rotas escolhidas pela vizinhança.

Devido à alta dinamicidade da rede em VANETs, alguns sistemas optam por empregar uma abordagem infraestruturada, assim eliminando a difícil tarefa de selecionar os veículos, dentro de um subconjunto, mais relevante para detectar congestionamentos e sugerir rotas alternativas. Uma outra solução para a abordagem distribuída é fazer segmentação do cenário [de Souza and Villas 2016] e escolher o veículo mais central dentro dela. No entanto essa escolha nem sempre é a mais adequada. Com o objetivo de aperfeiçoar as soluções existentes, o trabalho proposto apresenta um mecanismo de classificação baseado em agrupamento (clustering) dinâmico de veículos baseado na métrica de SNA e também adota dois conceitos de SNC (comunidade virtual e interação social) para a decisão do roteamento colaborativo.

\section{MAESTRO}

MAESTRO é um sistema distribuído de gerenciamento de mobilidade urbana baseado nas VSNs. O objetivo do sistema é aprimorar o fluxo veicular nas vias sem comprometer a escalabilidade do sistema. Para isso, o sistema é composto de três componentes: (i) coleta de dados; (ii) mecanismo de agrupamento para geração do conhecimento; e (iii) planejamento de rota colaborativo. A descrição detalhada de cada componente é apresentada a seguir.

\subsection{Coleta de Dados}

Crowdsensing móvel é um paradigma que utiliza o conceito de computação ubíqua na coleta de dados, além de compartilhar tais dados [Bazzi and Zanella 2016, Wang et al. 2018]. Além disso, por meio da agregação dos dados coletados, pode-se criar uma consciência local. Esta, por sua vez, pode auxiliar em inúmeras aplicações de larga escala, como por exemplo, monitoramento da poluição do ar e alertas de congestionamento de tráfego veicular. Sabe-se que nas VSNs, os veículos são equipados com tecnologias de computação e de comunicação sem-fio juntamente com sensores inteligentes, possibilitando assim a aplicação do paradigma de vehicle crowdsensing (VCS) [Wang et al. 2018]. Tal paradigma possibilita o monitoramento de fenômenos dinâmicos e em grande escala.

A motivação do uso do VCS está no fato de que os participantes da VSNs possam solucionar problemas em cooperação com os outros participantes da rede. Por exemplo, os participantes da VSNs podem conjuntamente aprimorar a mobilidade urbana por meio do compartilhamento dos dados coletados sobre as condições do tráfego veicular. Ao fazer isso, aplicações de VSNs podem agregar tais dados (consciência local) e gerar conhecimento sobre as condições de tráfego em tempo-real. O conhecimento gerado auxiliará o gerenciamento da mobilidade urbana.

Neste trabalho, para criar a consciência local do tráfego foi aplicado o paradigma de VCS. Para este fim, cada veículo, $n$, compartilha periodicamente mensagens curtas (beacon) de status $\left(b_{n}\right)$. Tais mensagens contêm dados sobre o veículo, como velocidade 
atual $\left(s_{n}\right)$, localização $\left(p_{n}\right)$, time $\operatorname{stamp}\left(t_{n}\right)$ e a pontuação do veículo $\left(v_{e s c_{n}}\right)$. Este último dado será utilizado no mecanismo de agrupamento que será explicado mais adiante.

\subsection{Mecanismo de Agrupamento para Geração do Conhecimento}

Um dos grandes desafios em redes altamente dinâmicas é selecionar de maneira distribuída os nós mais apropriados, dentro de um subconjunto, para realizar determinada tarefa. Com o objetivo de superar tal desafio, neste trabalho foi aplicada a técnica de agrupamento dinâmico. Diferente do sistema FASTER [de Souza and Villas 2016], MAESTRO não necessita previamente segmentar todo cenário para selecionar o veículo que realizará a tarefa de detecção de congestionamento.

No MAESTRO, cada agrupamento associa-se a um conjunto de veículos denominado de cluster member $(\mathrm{CM})$ e um representante nomeado de cluster head $(\mathrm{CH})$, conforme exibido na Figura 1. Na mesma figura, os veículos representados pelas letras $A$ e $B$ representam os $\mathrm{CHs}$ dos agrupamentos 1 e 2 , respectivamente, enquanto que os demais elementos representam os CMs. O CH é o veículo temporariamente selecionado com a responsabilidade de encaminhar os dados em nome dos vizinhos distantes de um salto de comunicação. O veículo que possui a maior pontuação $\left(v_{e s c_{n}}\right)$ é selecionado como $\mathrm{CH}$, os detalhes do cálculo da pontuação é dada a seguir. Por meio da técnica de agrupamento dinâmico, pode-se atacar os seguintes pontos: (i) selecionar o veículo mais apropriado de forma distribuída; (ii) minimizar a sobrecarga da rede; (iii) facilitar o fluxo de dados dentro da rede; e ( $i v)$ aumentar a escalabilidade do sistema.

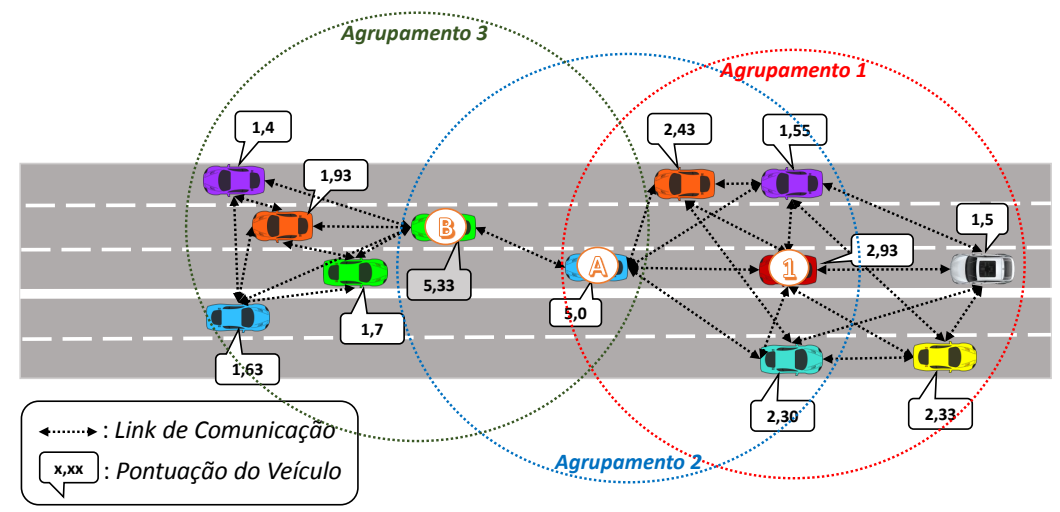

Figura 1. Agrupamento adaptativo dos veículos, nos quais os rótulos $A$ e $B$ representam os CHs temporários dos agrupamentos 1 e 2, respectivamente.

A ideia aqui é explorar as propriedades sociais dos nós da rede veicular, com intuito de melhorar o processo de difusão dos dados. Este deverá ser feito por um caminho com o mínimo de interferência na comunicação e sem sobrecarregar a rede. Para isso, cada veículo autonomamente calcula sua pontuação de acordo com os links de comunicação das vizinhanças, juntamente com um indicador de intensidade do sinal recebido, conforme exibido na Equação 1:

$$
v_{e s c_{n}}=\sum_{M_{n}(i, j) \neq 0, i<j} \frac{1}{M_{n}^{2}\left[1-M_{n}\right]_{i, j}}+\left(P L\left(d_{0}\right)+10 \alpha \log _{10}\left(\frac{d}{d_{0}}\right)+\Psi_{\sigma_{\text {single }}}\right), d \geq d_{0}
$$

A primeira metade da equação representa a métrica de redes egocêntricas, a qual foi escolhida pela vantagem de utilizar apenas as informações da topologia local para seu propósito. Mais especificamente, foi aplicada a métrica da centralidade de intermediação 
egocêntrica (CIE) [Akabane et al. 2017, Akabane et al. 2018a]. Esta métrica indica a influência do nó na difusão de dados dentro da rede. Sabe-se que a uma matriz de adjacência, $\left(M_{k \times k}\right)$, pode representar os canais de intercomunicação entre os nós, no qual $k$ é o número de nós de 1-salto de comunicação. O cálculo da CIE é dada pela soma inversa da equação $\left(M_{n}^{2}\left[1-M_{n}\right]_{i, j}\right)$, onde $M_{n}$ representa a matriz de adjacência do veículo $n$, $M_{n}^{2}$ indica a distância geodésica entre os pares de nós $i$ e $j$, por fim, 1 na expressão corresponde uma matriz com todos os elementos iguais a 1. A segunda metade da equação refere-se ao indicador de intensidade do sinal recebido. O modelo aplicado foi o logdistance path-loss [Nilsson et al. 2017], onde $d$ é a distância Euclidiana entre os veículos, $d_{0}$ é a distância de um ponto de referência até ao emissor, $P L\left(d_{0}\right)$ é a potência do ponto de referência até ao emissor, $\alpha$ representa o expoente do path-loss (varia de acordo com o ambiente) e $\Psi_{\sigma_{\text {single }}}$ é uma variável que descreve a atenuação do sinal de comunicação. Em poucas palavras, neste modelo a potência do sinal recebido decai logaritmicamente com a distância entre os veículos. Por meio da Equação 1, a cada mudança na topologia local é feita a atualização da pontuação do veículo. Esta informação é anexada no $v_{e s c} \mathrm{e}$ transmitida no beacon subsequente.

Antes de descrever os detalhes da agregação de dados para a geração do conhecimento, uma definição formal da topologia da rede viária é necessária.

Definição: A topologia da rede viária pode ser descrita por meio de um grafo direcionado $G=(V, E)$, no qual $V$ corresponde um conjunto de intersecções $(v)$, enquanto que $E$ corresponde um conjunto de segmentos de via (e, onde e $\in E \subseteq V^{2}$ ). Além disso, cada segmento de via é composto por um peso $(\rho)$ que indica o nível de serviço, que será explicado em detalhes mais adiante. Por fim, uma rota $r(A, B)$ entre dois pontos $A$ e $B$ é uma sequência de intersecções $\left(v_{1}, \ldots, v_{n}\right)$ tal que $v_{1}=A, v_{n}=B$ e todos os pares de intersecções consecutivos são conectados por um segmento de via, isto é, para todo $i=1, \ldots, n-1$ existe $\left(v_{i}, v_{i+1}\right) \in E$.

Para geração do conhecimento sobre a condição de tráfego veicular, necessita-se aplicar duas diferentes abordagens de agregação: (i) agregação dos beacons recebidos da vizinhança - consciência local (Equação 2) e (ii) agregação da consciência local conhecimento da condição de tráfego (Equação 3).

$$
\Lambda:=\left(E^{\prime}, \Upsilon, \Omega\right)
$$

onde $E^{\prime}=\left\{e_{1}, \ldots, e_{n}\right\} \mid E^{\prime} \in E(G)$. Os parâmetros $\Upsilon$ e $\Omega$ representam $\left\{t_{1}, \ldots, t_{n}\right\}$ e $\left\{v_{m_{1}}, \ldots, v_{m_{n}}\right\}$, ou seja, o tempo atual e velocidade média de cada elemento de $E^{\prime}$.

$$
\Lambda_{r, s}:=\sum_{1 \leq r, s \leq n} \sigma \Lambda_{r}+(1-\sigma) \Lambda_{s},\left\{\begin{array}{l}
t_{r}>t_{s} \\
s_{r}, s_{s} \neq 0
\end{array}\right.
$$

onde $\sigma$ é o fator de ponderação. A finalidade deste fator é considerar as informações mais atuais no processo de agregação $\left(t_{r}>t_{s}\right)$.

Retornando ao exemplo da Figura 1, supondo que o veículo (1) inicia o processo de geração da consciência local, uma vez terminado, ele repassa a informação agregada para o $\mathrm{CH}(A)$. O veículo $(A)$ realiza a agregação dos beacons da sua vizinhança (Equação 2) com a informação agregada recebida do veículo (1) (Equação 3) e repassa o resultado das agregações para o $\mathrm{CH}$ subsequente $(B)$ e assim por diante. Neste exemplo ilustrativo, o $(B)$ possui a maior pontuação temporariamente, logo este é encarregado de computar o peso de cada via de acordo com a Equação 4:

$$
\rho_{k}=v_{a g r_{k}}^{\text {med }} \times\left(1-v_{e_{k}}^{l i m}\right)^{-1} \mid \forall e_{k} \in E^{\prime}
$$


onde os parâmetros $v_{a g r_{k}}^{\text {med }} v_{e_{k}}^{\text {lim }}$ representam a velocidade média agregada e a velocidade máxima permitida na via, $k$, respectivamente.

Terminada essa etapa, o $(B)$ classifica o peso de acordo com o nível de serviço (LOS - Level of Service), Tabela 1. Esta tabela apresenta a classificação do tráfego em cada nível de serviço de acordo com o peso ( $\rho$ ) do High Capacity Manual [Manual 2010]. Cada nível de serviço representa uma condição de tráfego veicular. Durante a etapa de classificação, caso sejam encontradas vias congestionadas, LOS $D, E$ e $F$, gera-se uma mensagem contendo informação de tais vias e inicia-se o processo de disseminação. Para evitar o problema de tempestade de broadcast durante o processo de disseminação, foi aplicado o conceito de zona de preferência (ZoP) [Akabane et al. 2015]. ZoP é uma região dentro do raio de transmissão, na qual veículos dentro dela são os mais apropriados para continuar o processo de disseminação. O conceito de ZoP é baseado em atraso, ou seja, os veículos dentro dela têm menor atraso (ou prioridade) do que os veículos fora dela. Assim, os veículos fora da ZoP recebem informações redundantes e cancelam a transmissão programada.

Tabela 1. Classificação de cada nível de serviço de acordo com $\rho$ [Manual 2010].

\begin{tabular}{ccc}
\hline Nível de serviço & Classificação do tráfego & Peso da via $(\rho)$ \\
\hline A & Tráfego livre & $(0,0 \sim 0,33]$ \\
B & Tráfego razoavelmente livre & $(0,33 \sim 0,4]$ \\
C & Tráfego Moderado & $(0,4 \sim 0,5]$ \\
D & Congestionamento leve & $(0,5 \sim 0,7]$ \\
E & Congestionamento crítico & $(0,7 \sim 0,9]$ \\
F & Tráfego totalmente colapsado & $(0,9 \sim 1,0]$
\end{tabular}

\subsection{Planejamento de Rota Colaborativo}

Conforme mencionado anteriormente, as VSNs envolvem interações sociais dentro de uma comunidade virtual temporal de veículos com base em interesses em comum ou objetivos mútuos [Vegni and Loscri 2015, Rahim et al. 2017]. Inspirado nessa ideia foi proposto o planejamento de rota colaborativo aplicando dois conceitos de SNC: comunidade virtual temporal e interações sociais, Figura 2. Assim, todos os veículos dentro da área da comunidade virtual temporal são considerados participantes dela. As interações sociais entre os participantes da comunidade são feitas por meio de canais de comunicação V2V e as informações trocadas (de interesse em comum) são as rotas alternativas escolhidas. Lembrando que a área coberta pela comunidade virtual depende do raio da circunferência definida pela aplicação e o ponto central da área da comunidade é o local do congestionamento detectado. O objetivo principal é rotear a máxima quantidade de veículos para contornar os congestionamentos, ao longo de sua rota, sem causar congestionamento secundários.

Para isso, durante a fase de planejamento de rota, os veículos dentro da comunidade virtual temporal e mais próximos do local de congestionamento têm a prioridade na escolha de uma rota alternativa. Antes de calcular uma rota alternativa, o veículo computa a popularidade ( $p o p)$ das vias de acordo com as rotas alternativas escolhidas pelos veículos da vizinhança. O objetivo da pop é sinalizar as vias mais escolhidas/populares para contornar o congestionamento. Assim, a popularidade da via $(v)$ é dada: $\operatorname{pop}_{v}=\operatorname{num}_{v} \times\left(\operatorname{comp}_{(v)} / \operatorname{lin}_{(v)}\right)$, onde $\operatorname{num}_{v}, \operatorname{comp}_{(v)}$ e $\operatorname{lin}(v)$ representam a quantidade de veículos, o comprimento da via e a quantidades de linhas, respectivamente.

Considere que $R\left(P_{\text {atual }}, D_{\text {est }}\right)$ representa o conjunto de todas as possíveis rotas alternativas da posição atual $\left(P_{\text {atual }}\right)$ até o destino $\left(D_{\text {est }}\right)$. Assim, a escolha de uma rota alternativa $r^{*}$ é dada pela Equação 5, ou seja, o veículo seleciona a rota menos popular 


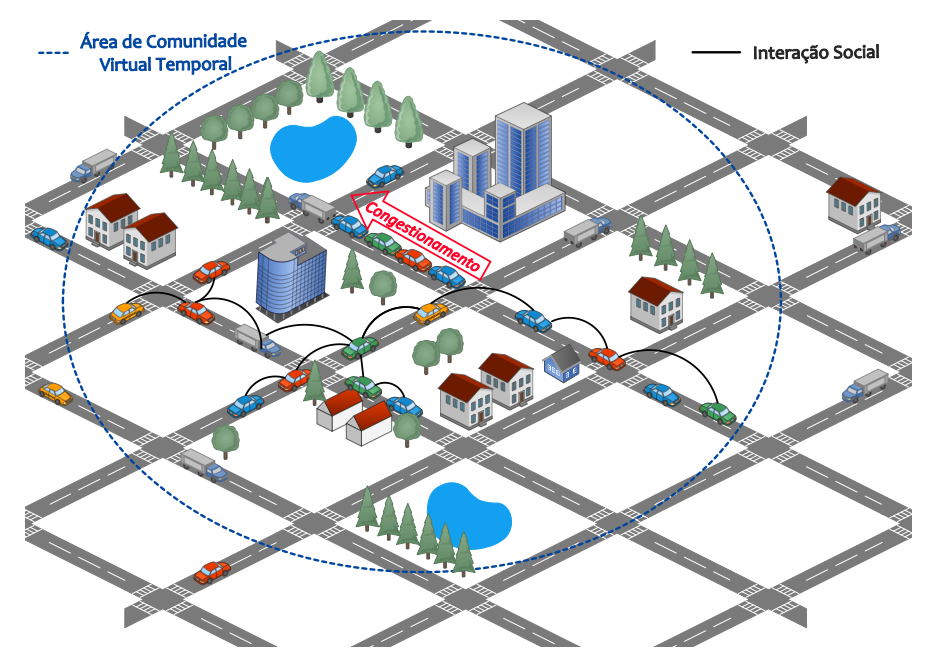

Figura 2. Exemplo ilustrativo da interações sociais e área de comunidade virtual temporal em VSNs.

entre todas as possíveis rotas e a compartilha por meio da interação social. Dessa forma, reduzindo a possibilidade de gerar congestionamento em outro local futuramente.

$$
r^{*}\left(P_{\text {atual }}, D_{\text {est }}\right)=\underset{r \in R\left(P_{\text {atual }}, D_{\text {est }}\right)}{\operatorname{menosPopular}}\|r\|
$$

\section{Avaliação de Desempenho}

Nesta seção é apresentada a metodologia científica aplicada neste trabalho, os simuladores utilizados e também os resultados comparativos do sistema proposto com outros três sistemas da literatura.

\subsection{Simuladores e Configurações dos Experimentos}

A avaliação de desempenho do sistema MAESTRO foi conduzida por meio de simulações. Uma das ferramentas utilizadas foi o Veins 4.5 - Vehicular Network Simulations $^{1}$. Esta integra o simulador OMNet++ 5.0 - Network Simulation Framework ${ }^{2}$ com o SUMO 0.29.0 - Simulation of Urban MObility ${ }^{3}$. Vale ressaltar que o Handbook Emission Factors for Road Transport ${ }^{4}$ (HBEFA) foi aplicado para medir a emissão de $\mathrm{CO}_{2}$.

Para garantir que o sistema de gerenciamento de mobilidade urbana proposto seja avaliado em um cenário próximo da vida real, foi utilizado o projeto TAPASCologne ${ }^{5}$. Tal projeto visa reproduzir as mobilidades dos veículos de forma mais realista possível da cidade de Köln, Alemanha. O projeto utilizado contêm 250.484 rotas de veículos que descrevem a demanda real de duas horas toda a região demarcada. No entanto, apenas uma subregião central (46.596 rotas) foi considerada nos experimentos das simulações, pois ela revela uma maior incidência de congestionamento de tráfego (LOS $D, E$ e $F$ ), conforme destacado na Figura 3 e também na barra de calor. Além disso, com esse novo conjunto de dados foram criados cinco diferentes conjuntos, de forma incremental, que representam as taxas de inserção de veículos $(20 \%, 40 \%, 60 \%, 80 \%$ e $100 \%)$ para os

\footnotetext{
${ }^{1}$ http://veins.car2x.org/

${ }^{2}$ https://omnetpp.org/

${ }^{3} \mathrm{http}: / /$ sumo.sourceforge.net/

${ }^{4} \mathrm{http}: / /$ www.sumo.dlr.de/wiki/Models/Emissions/HBEFA-based

${ }^{5} \mathrm{http} / / /$ kolntrace.project.citi-lab.fr/
} 
experimentos. Isso significa que, do total do novo conjunto de dados, apenas $20 \%$ dos veículos são inseridos no cenário para os experimentos de simulação, e assim por diante.

Cada veículo foi configurado com uma potência de transmissão de $20 \mathrm{~mW}$, bitrate de $6 \mathrm{Mbps}$ e o raio de comunicação de $287 \mathrm{~m}$. O padrão de comunicação utilizado foi o IEEE 802.11p. Por fim, a Tabela 2 descreve os principais parâmetros de simulação aplicados nos experimentos.

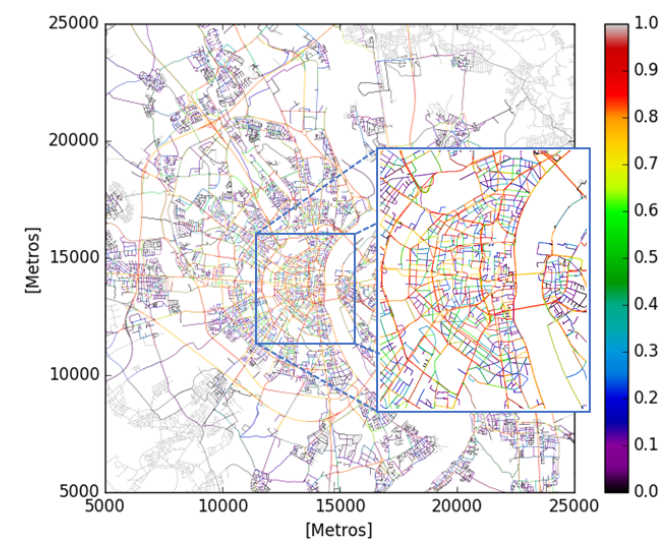

Tabela 2. Parâmetros.

\begin{tabular}{ll}
\hline Parâmetro & Valor \\
\hline \hline Taxa de inserção de veículos & $20 \%$ a $100 \%$ \\
Camada MAC & $802.11 \mathrm{p}$ \\
Potência de transmissão & $20 \mathrm{~mW}$ \\
Bitrate & $6 \mathrm{Mbps}$ \\
Sensibilidade de recepção & $-82 \mathrm{dBm}$ \\
Raio de transmissão & $287 \mathrm{~m}$ \\
Frequência de transmissão (Beacon) & $1 \mathrm{~Hz}$ \\
Raio da comunidade temporal virtual & $1 \mathrm{Km}$ \\
Intervalo de confiança & $95 \%$ \\
\hline
\end{tabular}

Figura 3. Cenário de simulação.

\subsection{Avaliação dos resultados das simulações}

As avaliações dos resultados foram divididas em duas etapas. Na primeira etapa foi avaliada a escalabilidade dos sistemas, Subseção 4.2.1. Na segunda etapa, Subseção 4.2.2, foi avaliado o gerenciamento da mobilidade urbana.

\subsubsection{Avaliação de Escalabilidade}

Com o intuito de avaliar a escalabilidade do sistema MAESTRO, juntamente com os sistemas EcoTrec [Doolan and Muntean 2017], FASTER [de Souza and Villas 2016] e DIVERT [Pan et al. 2017], os experimentos de simulações foram realizados variando as taxas de inserção dos veículos de $20 \%$ a $100 \%$ da subregião selecionada. Adicionalmente, as métricas de avaliação aplicadas foram: (i) Mensagens transmitidas: indica o total de mensagens transmitidas para realizar a entrega do conhecimento gerado; (ii) Colisões de pacote: exibe o número total de colisões de pacotes durante a transmissão das mensagens; (iii) Latência: aponta o tempo gasto para realizar a entrega das mensagens para os veículos; e (iv) Cobertura: expõe a porcentagem de mensagens entregue para os veículos. A Figura 4 apresenta os resultados em termos de escalabilidade. Cada subfigura apresentada possuí uma tabela com valores numéricos da métrica avaliada, juntamente, com o gráfico de barras que representa o ganho em relação ao EcoTrec.

A Figura 4(a) apresenta os resultados do desempenho dos sistemas investigados de acordo com a métrica de overhead. Sabe-se que os sistemas EcoTrec e DIVERT necessitam constantemente trocar mensagens entre veículo e servidor para alcançar seus objetivos. Devido a essa abordagem, pode-se observar na tabela que ambos possuem maior taxa média de mensagens transmitidas em relação ao MAESTRO e FASTER. Outra consequência direta dessa alta taxa, para ambos sistemas, é a ausência de um mecanismo de supressão de broadcast durante o processo de distribuição de mensagens. Também é possível observar, na mesma tabela, que DIVERT possui uma taxa de transmissão ligeiramente maior do que EcoTrec. Isto se deve ao fato de que o DIVERT, além da comunicação com o servidor, aplica o mecanismo de roteamento colaborativo na escolha de uma rota 


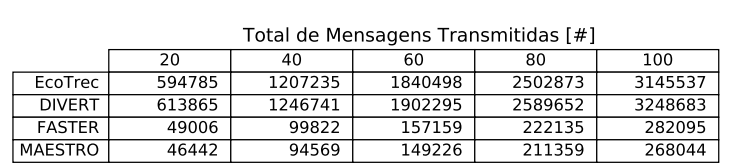

Ganho na Taxa de Transmissão em Relação ao EcoTrec [\%]

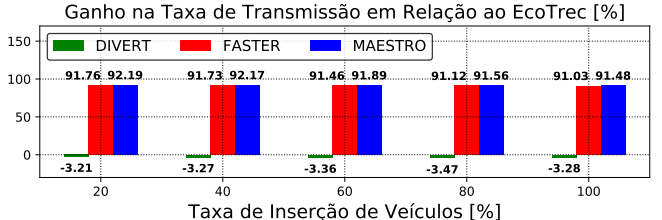

(a) Total de mensagens transmitidas.

\begin{tabular}{|c|c|c|c|c|c|}
\hline & \multicolumn{5}{|c|}{ Latência [segundos] } \\
\hline & 20 & 40 & 60 & 80 & 100 \\
\hline EcoTrec & 1.16 & 1.50 & 1.88 & 2.21 & 2.50 \\
\hline DIVERT & 1.24 & 1.59 & 1.94 & 2.26 & 2.55 \\
\hline FASTER & 0.30 & 0.34 & 0.39 & 0.43 & 0.57 \\
\hline MAESTRO & 0.35 & 0.40 & 0.45 & 0.50 & 0.64 \\
\hline
\end{tabular}

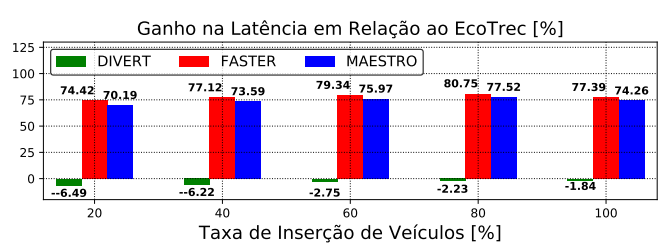

(c) Latência.

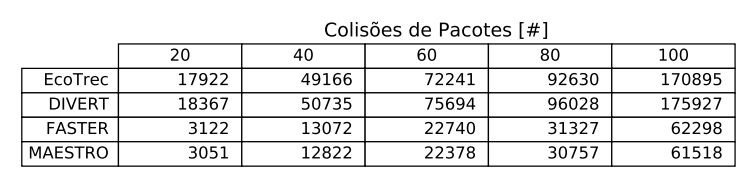

Ganho na Taxa Colisão em Relação ao EcoTrec [\%]

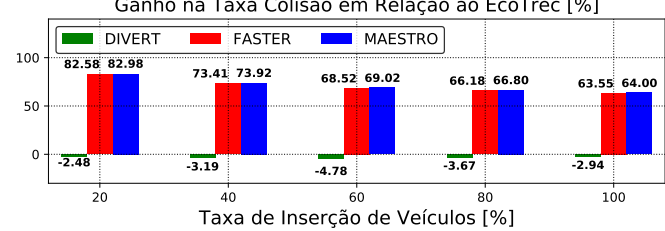

(b) Colisões de pacotes.
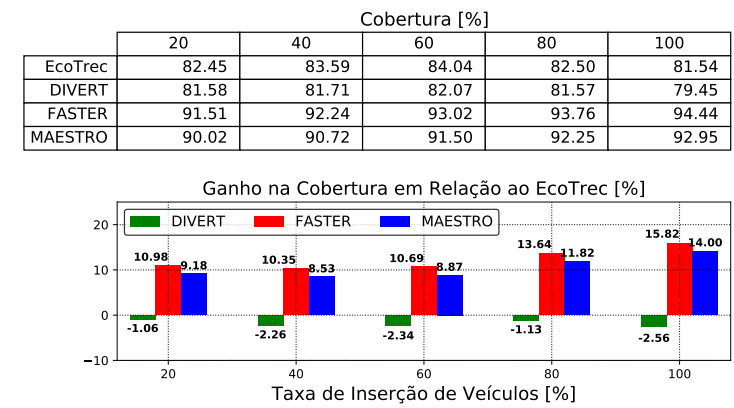

(d) Cobertura.

Figura 4. Resultados da avaliação de escalabilidade.

alternativa. Vale a pena mencionar que tal mecanismo contribui no gerenciamento do tráfego e essa contribuição será discutida na subseção a seguir. Tanto MAESTRO quanto FASTER aplicam técnica de seleção de veículos para a geração de conhecimento. $\mathrm{O}$ primeiro segmenta o cenário em várias subregiões e em cada uma delas é selecionado o veĩculo para geração do conhecimento. O segundo aplica a técnica de agrupamento dinâmico para selecionar o veículo mais adequado. O agrupamento dinâmico tem a vantagem de não necessitar, previamente, segmentar o cenário para a seleção do veículo. Vale salientar que, MAESTRO e o FASTER aplicam um mecanismo para lidar com o problema da tempestade de broadcast. Observa-se que MAESTRO e FASTER possuem desempenho similar e ambos são capazes de reduzir drasticamente a quantidade total de mensagens transmitidas, acima de $91 \%$ em comparação com DIVERT e EcoTrec, como apresentado na figura de barras.

A Figura 4(b) exibe a quantidade de colisões de pacotes em função da taxa de inserção de veículos. Sabe-se que os sistemas EcoTrec e DIVERT possuem a maior sobrecarga da rede em comparação com MAESTRO e FASTER, e a consequência direta disso é uma maior quantidade de colisões de pacotes, como observada na tabela. Como MAESTRO e FASTER possuem baixa sobrecarga na rede entre os competidores, consequentemente possuem também menores taxas de colisão de pacotes. A porcentagem de redução alcançada, para ambos, são em torno de 70,8\% e 74,2\% para toda taxa de inserção de veículos, comparado com os sistemas EcoTrec e DIVERT, respectivamente, como exibido na figura de barras.

Outra métrica avaliada é a latência de transmissão em relação à taxa de inserção de veículos, Figura 4(c). Tanto na abordagem infraestruturada quanto distribuída, à medida que as taxas de inserção de veículos aumentam a latência também aumenta, como esperado. Isso se deve ao fato que aumentando a quantidade de veículos no cenário, aumenta-se o overhead causado na rede. No entanto, MAESTRO e FASTER possuem as menores latências em comparação com outros sistemas analisados. Comparando nu- 
mericamente, o atraso médio dos sistemas MAESTRO, FASTER, DIVERT e EcoTrec é de $0,47 \mathrm{~s}, 0,40 \mathrm{~s}, 1,91 \mathrm{~s} \mathrm{e} 1,85 \mathrm{~s}$, respectivamente. O sistema FASTER possui uma ligeira redução na latência em comparação ao MAESTRO, devido ao fato de que os conhecimentos são gerados em várias subregiões, assim entregando-os mais rapidamente para os veículos. Tanto MAESTRO quanto FASTER têm uma redução média acima de $74 \% \mathrm{em}$ comparação com os sistemas EcoTrec e DIVERT, como mostrado na figura de barras.

A Figura 4(d) demonstra a cobertura atingida em função da taxa de inserção de veículos. A partir da tabela, pode-se observar que o EcoTrec tem uma cobertura ligeiramente maior que o DIVERT. Isto se deve ao fato do EcoTrec possuir uma menor sobrecarga na rede, Figuras 4(a) e 4(b), quando comparado com seu oponente. Na mesma tabela, pode-se verificar em altas taxas de inserção (80\% e $100 \%$ ), EcoTrec e DIVERT possuem uma ligeira redução na cobertura. Pois nesse intervalo a rede torna-se muito densa e consequentemente elevando a concorrência no acesso ao canal, além de aumentar a quantidade de colisões de pacotes. Por outro lado, como os sistemas MAESTRO e FASTER apresentam menores sobrecargas da rede, os conhecimentos gerados por eles podem atingir uma quantidade maior de veículos em todas as taxas de inserção analisadas, como observado na tabela. FASTER apresenta um resultado ligeiramente superior em relação ao MAESTRO, devido ao fato de que os conhecimentos são gerados em várias subregiões, assim alcançando uma maior cobertura.

\subsubsection{Avaliação do Gerenciamento da Mobilidade Urbana}

Nesta seção foi avaliado o gerenciamento da mobilidade urbana do sistema MAESTRO, comparando-o com os outros três sistemas anteriormente mencionados. Além disso, as métricas de avaliações aplicadas foram: (i) Tempo médio de viagem: denota o tempo médio de viagem de todos os veículos, ou seja, indica o estado geral do tráfego para toda a rede viária observada; (ii) Tempo perdido em congestionamento: expõe o tempo médio perdido em congestionamento; (iii) Índice de tempo de viagem: mede o nível de congestionamento do tráfego urbano [Schrank et al. 2012]. Tal índice é calculado pela razão entre o tempo total de viagem e o tempo de viagem em fluxo livre. A última métrica avaliada é a (iv) Emissão de $\mathrm{CO}_{2}$ : indica a emissão média de $\mathrm{CO}_{2}$ de todos os veículos que estão no cenário. A Figura 5 apresenta os resultados obtidos de acordo com cada métrica. Cada subfigura é composta por dois gráficos de barras, o superior representa os valores numéricos da métrica avaliada, e o inferior simboliza o ganho em relação ao EcoTrec.

A Figura 5(a) apresenta o resultado do tempo médio de viagem para todas as taxas de inserção. A partir da figura, observa-se que, quanto maior a taxa de inserção, maior é o tempo médio de viagem para todas as soluções analisadas. Esse comportamento é esperado uma vez que, em elevadas taxas, as vias ficam mais densas, levando à ocorrência de congestionamentos. Dentre as soluções analisadas, EcoTrec apresenta o maior tempo médio de viagem, em torno de 22 minutos. Sabe-se que a escolha de uma rota alternativa em tal sistema é dada pelo caminho que emite a menor taxa de $\mathrm{CO}_{2}$ até o destino. O sistema FASTER seleciona uma rota, de forma egoísta, com base no algoritmo de $k$ menores caminhos com escolha probabilística. Observa-se que essa estratégia possui um ganho de 6,82\%, em relação ao EcoTrec. No sistema DIVERT, os veículos calculam uma rota alternativa de maneira colaborativa. Em tal abordagem é possível notar uma redução no tempo médio de viagem em torno de $15 \%$ e $8,3 \%$, comparado com o EcoTec e FASTER, respectivamente. O sistema MAESTRO aplica o roteamento colaborativo, como DIVERT. Mesmo assim ele supera o DIVERT em tal métrica, devido à baixa sobrecarga na rede. Como se sabe, DIVERT possui uma alta sobrecarga, logo muitas mensagens chegam nos destinatários de forma corrompida. Analisando numericamente, MAESTRO atinge uma redução média de 21,45 \%, 14,74 \% e 6,42 \% em comparação com o EcoTrec, 


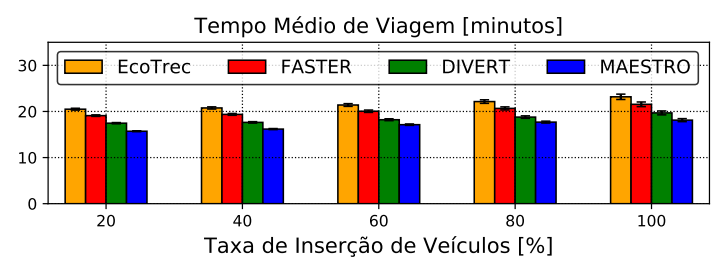

Ganho no Tempo de Viagem em Relação ao EcoTrec [\%]

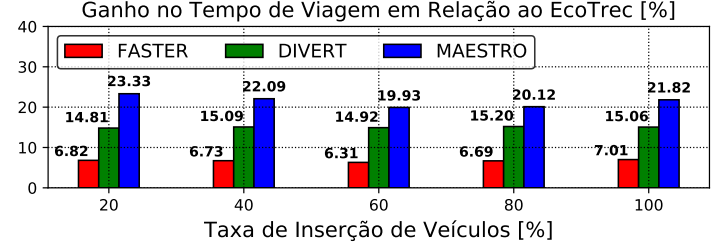

(a) Tempo médio de viagem.

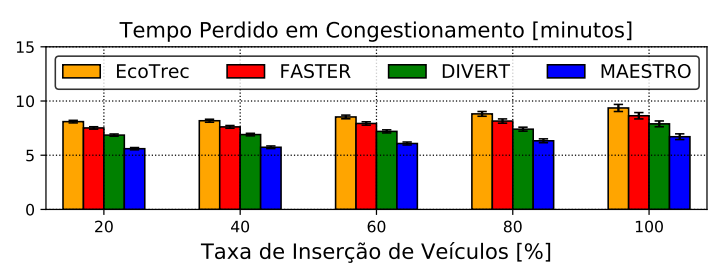

Ganho no Tempo Perdido em Relação ao EcoTrec [\%]

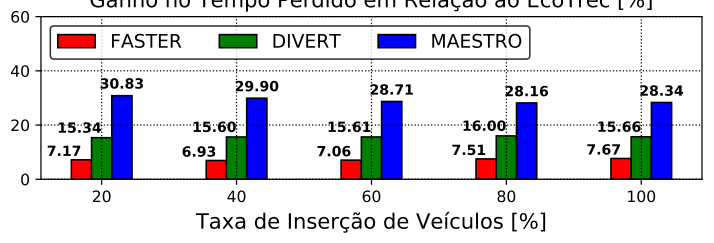

(c) Tempo perdido em congestionamento.

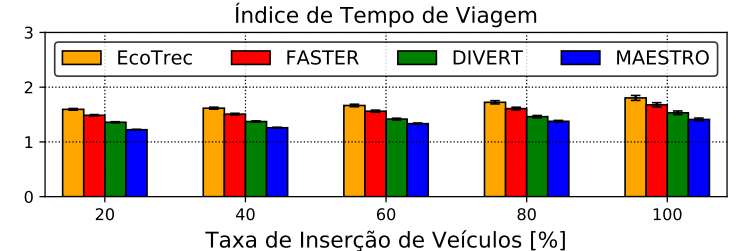

Ganho no Índice de Tempo em Relação ao EcoTrec [\%]

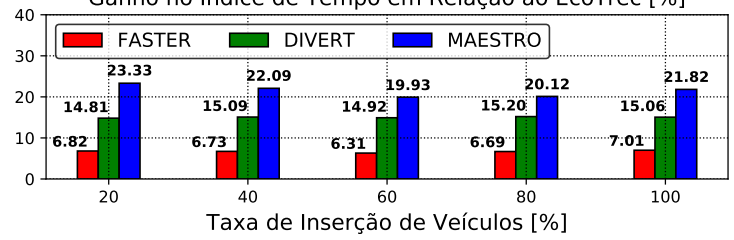

(b) Índice de tempo de viagem.

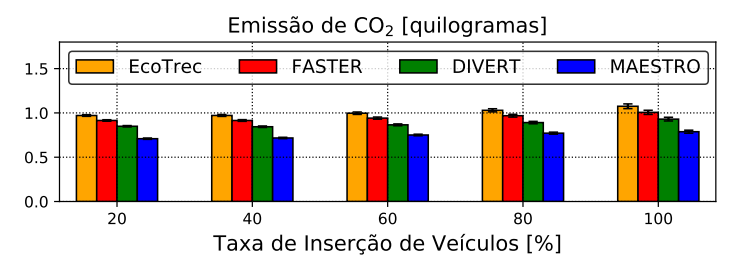

Ganho na Emissão de $\mathrm{CO}_{2}$ em Relação ao EcoTrec [\%]

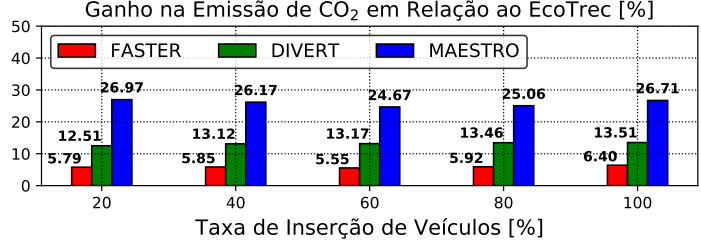

(d) Emissão de $\mathrm{CO}_{2}$

Figura 5. Resultados da avaliação do gerenciamento da mobilidade urbana.

FASTER e DIVERT, respectivamente.

A Figura 5(b) indica o nível de congestionamento de tráfego em função da taxa de inserção de veículos. Observa-se que os resultados dessa métrica apresentam comportamentos similares à métrica do tempo médio de viagem (Figura 5(a)). Isso se deve pelo fato que ambas as métricas levam em consideração o tempo médio de viagem. Como discutido anteriormente, o sistema DIVERT possui uma ligeira sobrecarga na rede em relação ao EcoTrec, pois há trocas de informações de rotas alternativas entre as vizinhanças. Entretanto, essa ligeira sobrecarga, faz com que o DIVERT supere o seu oponente nas métricas de tempo de viagem, índice de tempo de viagem, além de outras duas métricas a seguir.

Outra métrica importante a ser avaliada é o tempo perdido em congestionamento, Figura 5(c). Todos os sistemas avaliados aplicam algum mecanismo de roteamento de veículos após a detecção do congestionamento. É importante ressaltar que essa métrica deixa clara a vantagem do uso do roteamento colaborativo em relação ao egoísta. Isto acontece pois aqueles que a aplicam possuem o menor tempo desperdiçado no trânsito. Para demonstrá-los numericamente, DIVERT atinge uma redução de tempo de aproximadamente 15,64 \% em relação ao EcoTrec, enquanto o MAESTRO atinge aproximadamente 29,18\% comparado com EcoTrec. Como mencionado anteriormente, o sistema MAESTRO possui menor sobrecarga em comparação ao DIVERT. Logo, este fato contribui para que a informação alcance o maior número de participantes e, assim, contribuindo no gerenciamento do tráfego.

A Figura 5(d) demonstra a emissão de $\mathrm{CO}_{2}$ em função da taxa de inserção de veículos. Como esperado, EcoTrec apresenta a maior emissão de $\mathrm{CO}_{2}$ em todas as taxas 
de inserção analisadas, pois ele possui o maior índice de tempo de viagem (Figura 5(b)) e tempo perdido em congestionamento (Figura 5(c)). Por meio dessa métrica, pode-se notar que os sistemas mais eficientes no gerenciamento de mobilidade de urbana apresentam menor quantidade de emissão de $\mathrm{CO}_{2}$. Analisando numericamente, os sistemas FASTER, DIVERT e MAESTRO apresentaram uma redução média na emissão de $\mathrm{CO}_{2}$, em relação ao EcoTrec, de aproximadamente 5,9\%,13,15\% e 25,91\%, respectivamente.

\subsubsection{Considerações Finais}

Os resultados dos experimentos desse trabalho demonstraram que a dificuldade de selecionar o veículo mais adequado de forma distribuída, em redes altamente dinâmicas, pode ser contornado pelo mecanismo de agrupamento dinâmico com o apoio da SNA, mais especificamente a CIE. A principal vantagem desse mecanismo é utilizar somente o conhecimento local da topologia da rede para alcançar o seu objetivo, além de aumentar a capacidade de escalabilidade do sistema. As desvantagens desse mecanismo, em relação a outra solução distribuída que segmenta todo o cenário previamente são: ( $i$ ) menor cobertura e (ii) uma latência ligeiramente maior. Outra observação feita é que a tomada de decisão colaborativa é mais eficiente do que a egoísta no cálculo das rotas alternativas. Em resumo, as soluções distribuídas analisadas tende a ser mais escaláveis que a infraestruturadas e aquelas que adotam a estratégia do roteamento colaborativo tende ser mais eficiente no gerenciamento da mobilidade urbana.

\section{Conclusão}

É cada vez mais evidente a necessidade de um sistema eficiente de gestão da mobilidade urbana com o intuito de aprimorar o gerenciamento do tráfego veicular. Com o objetivo de atender esta demanda foi proposto o MAESTRO, um sistema distribuído de gerenciamento de mobilidade urbana baseado nas VSNs. A principal vantagem do MAESTRO é o uso combinado de duas abordagens das VSNs, tal como análise e conceitos de redes sociais. Uma métrica de análise de redes sociais, mais especificamente, a métrica de centralidade de intermediação egocêntrica foi empregada para atuar como mecanismo de classificação de veículos. Além disso, dois conceitos de redes sociais foram empregados para a estratégia de roteamento colaborativo, neste caso, a interação social e a comunidade virtual temporal. Um conjunto de experimentos de simulação foi realizado por meio de duas perspectivas: (i) avaliação de escalabilidade e (ii) avaliação do gerenciamento da mobilidade urbana. O sistema proposto foi comparado com três sistemas da literatura, EcoTrec, FASTER e DIVERT. Com base nas análises dos resultados dos experimentos, pode-se concluir que o MAESTRO é um sistema de gerenciamento de mobilidade urbana eficiente e escalável.

Como trabalho futuro, pretende-se incorporar os padrões de mobilidade dos motoristas e preferências de usuários no sistema MAESTRO para o planejamento das rotas alternativas.

\section{Agradecimentos}

Este trabalho foi parcialmente financiado pelas bolsas 2015/25588-6 e 2018/02204-6 da Fundação de Amparo à Pesquisa do Estado de São Paulo (FAPESP), pelo Conselho Nacional de Desenvolvimento Científico e Tecnológico (CNPq 401802/2016-7), e pela Coordenação de Aperfeiçoamento de Pessoal de Nível Superior - Brasil (CAPES) - Código de Financiamento 001. Este trabalho faz parte do projeto INCT denominado Internet do Futuro para Cidades Inteligentes (CNPq 465446/2014-0, CAPES 88887.136422/2017-00 e FAPESP 2014/50937-1). 


\section{Referências}

Akabane, A., Immich, R., Pazzi, R., Madeira, E., and Villas, L. (2018a). Distributed egocentric betweenness measure as a vehicle selection mechanism in vanets: A performance evaluation study. Sensors (Basel, Switzerland), 18(8).

Akabane, A. T., Immich, R., Madeira, E. R., and Villas, L. A. (2018b). imob: An intelligent urban mobility management system based on vehicular social networks. In 2018 IEEE Vehicular Networking Conference (VNC), pages 1-8. IEEE.

Akabane, A. T., Pazzi, R. W., Madeira, E. R., and Villas, L. A. (2017). Applying egocentric betweenness measure in vehicular ad hoc networks. In Network Computing and Applications (NCA), 2017 IEEE 16th International Symposium on, pages 1-4. IEEE.

Akabane, A. T., Villas, L. A., and Madeira, E. R. M. (2015). An adaptive solution for data dissemination under diverse road traffic conditions in urban scenarios. In Wireless Communications and Networking Conference (WCNC), pages 1654-1659. IEEE.

Bazzi, A. and Zanella, A. (2016). Position based routing in crowd sensing vehicular networks. Ad Hoc Networks, 36:409-424.

de Souza, A. M. and Villas, L. A. (2016). A fully-distributed traffic management system to improve the overall traffic efficiency. In Proceedings of the 19th ACM International Conference on Modeling, Analysis and Simulation of Wireless and Mobile Systems, pages 19-26. ACM.

Doolan, R. and Muntean, G.-M. (2017). Ecotrec-a novel vanet-based approach to reducing vehicle emissions. IEEE Transactions on Intelligent Transportation Systems, 18(3):608-620.

Manual, H. C. (2010). Hcm2010. Transportation Research Board, National Research Council, Washington, DC.

Nilsson, M. G., Gustafson, C., Abbas, T., and Tufvesson, F. (2017). A measurementbased multilink shadowing model for $\mathrm{v} 2 \mathrm{v}$ network simulations of highway scenarios. IEEE Transactions on Vehicular Technology, 66(10):8632-8643.

Pan, J. S., Popa, I. S., and Borcea, C. (2017). Divert: A distributed vehicular traffic rerouting system for congestion avoidance. IEEE Transactions on Mobile Computing, 16(1):58-72.

Qin, J., Zhu, H., Zhu, Y., Lu, L., Xue, G., and Li, M. (2016). Post: Exploiting dynamic sociality for mobile advertising in vehicular networks. IEEE Transactions on Parallel and Distributed Systems, 27(6):1770-1782.

Rahim, A., Kong, X., Xia, F., Ning, Z., Ullah, N., Wang, J., and Das, S. K. (2017). Vehicular social networks: A survey. Pervasive and Mobile Computing.

Schrank, D., Eisele, B., and Lomax, T. (2012). Tti's 2012 urban mobility report: Powered by inrix traffic data. college station, texas: Texas a\&m transportation institute, texas a\&m university system. accessed 18 april 2012.

Vegni, A. M. and Loscri, V. (2015). A survey on vehicular social networks. IEEE Communications Surveys \& Tutorials, 17(4):2397-2419.

Wang, S., Djahel, S., Zhang, Z., and McManis, J. (2016). Next road rerouting: A multiagent system for mitigating unexpected urban traffic congestion. IEEE Transactions on Intelligent Transportation Systems, 17(10):2888-2899.

Wang, X., Ning, Z., Hu, X., Ngai, E. C.-H., Wang, L., Hu, B., and Kwok, R. Y. (2018). A city-wide real-time traffic management system: Enabling crowdsensing in social internet of vehicles. IEEE Communications Magazine, 56(9):19-25. 